Methods The diagnosis of LGV was made following molecular diagnostic testing of an anal swab.

Results The patient presented as an emergency with a history of change in bowel habit, tenesmus and rectal bleeding. He had a past medical history of duodenitis and a family history of Crohn's disease. Digital rectal exam revealed a circumferential rectal tumour, $2 \mathrm{~cm}$ from the anal verge. Features suggested a diagnosis of rectal cancer and radiological staging demonstrated extensive local infiltration and nodal involvement, supporting this diagnosis. Biopsies from colonoscopy however revealed severe proctitis with no evidence of malignancy. The local colorectal MDT meeting decided the patient would have neoadjuvant chemoradiotherapy and subsequent surgery based on response, after more biopsies. In the interim he presented with pending bowel obstruction resulting in a de-functioning colostomy and the patient tested positive for HIV prompting a referral to GUM physicians. Repeat MRIs captured the subsequent remarkable response to LGV treatment with Doxycycline.

Discussion/conclusion It is important for HIV testing to be incorporated as part of the management plan for colorectal malignancies.

\section{CC4 OCULAR SYPHILIS ON THE RISE: A CASE SERIES}

${ }^{1}$ Clare Wood*, ${ }^{2}$ Jane Wells, ${ }^{2}$ Nick Jones, ${ }^{1}$ Ashish Sukthankar. ${ }^{1}$ Manchester Royal Infirmary, Manchester, UK; ${ }^{2}$ Manchester Royal Eye Hospital, Manchester, UK

\subsection{6/sextrans-2016-052718.43}

Background Ocular involvement of syphilis remains relatively rare, however our clinic has seen a recent flurry of cases with 13 new diagnoses in the last 2 years, compared with 11 seen in the proceeding 10 years. It can be difficult to diagnose with no pathognomonic signs and can affect any structure of the eye.

Aim To present a cluster of 13 new cases ocular syphilis diagnosed from 2013 until January 2016.

Methods A retrospective case review.

Results In conjunction with our tertiary eye hospital, our clinic saw 13 patients diagnosed with ocular syphilis between July 2013 and January 2016. All 13 patients were male: 6 heterosexual; 5 men who have sex with men (MSM) and 2 bisexual. 3 patients were HIV positive. Mean age 42 (range 22-75). Ocular involvement included uveitis (anterior, posterior and pan-), optic neuritis, papillitis and retinitis. Cases include both unilateral and bilateral symptoms. All were treated as per national guidelines for neurosyphilis with procaine penicillin plus probenecid, proceeded by oral steroids. The majority of these patients' symptoms resolved following treatment, however a few continue to have ongoing visual disturbances.

Discussion We present our 13 cases of ocular syphilis. They illustrate the diverse range of presentations of ocular syphilis and the importance of partnership between the GU clinic and specialist ophthalmology services.
CC5 A CASE OF URTICARIAL VASCULITIS LEADING TO HIV DIAGNOSIS

${ }^{1}$ Anna Garner, ${ }^{2}$ Hayley Colton*, ${ }^{2}$ Rokiah Ali, ${ }^{2}$ Sarah Cockayne. ${ }^{1}$ Stockport NHS Foundation Trust, Stockport, UK; ${ }^{2}$ Sheffield Teaching Hospitals NHS Foundation Trust, Sheffield, UK

\subsection{6/sextrans-2016-052718.44}

This abstract has been temporarily removed while important corrections are made.

\section{CC6 WARNINGS ARE NOT ENOUGH - A CASE SERIES OF RITONAVIR INDUCED CUSHING'S SYNDROME AND ADRENO-CORTICAL FAILURE}

${ }^{1}$ Isabel Reicher*, ${ }^{1,2}$ Daniel Richardson, 'Deborah Williams. ${ }^{1}$ Brighton \& Sussex medical School, Brighton, UK; ${ }^{2}$ Brighton \& Sussex University NHS hospitals Trust, Brighton, UK

\subsection{6/sextrans-2016-052718.45}

Background Ritonavir is a potent inhibitor of the cytochrome P450 3A4 enzyme used to boost other protease inhibitors in the management of HIV infection. The metabolism of fluorinated steroids (eg fluticasone, triamcinolone), used for asthma, hay fever and arthritis, is inhibited by ritonavir causing increased exposure to corticosteroid. Cases of ritonavir induced Cushing's syndrome and subsequent adrenocortical suppression were first reported in 1999. Despite awareness of this interaction new cases continue to occur.

Aim To identify and describe patients in our cohort with iatrogenic Cushing's \pm adrenocortical suppression, and to investigate whether there were missed opportunities for prevention.

Methods Cases were identified from laboratory and pharmacy records between January 2010 and December 2015. Data was collected on demographics, steroid use, presentation and outcome. GP and referral letters were reviewed.

Results 25 cases were identified. The steroids were prescribed in many different specialties, most commonly primary care and rheumatology, as well as being obtained OTC. Duration of steroid use ranged from a single dose to 3 injections over one year. The most common presentation was weight gain, facial swelling, fatigue and postural dizziness. Long-term sequelae included diabetes, osteoporosis and avascular necrosis as well as creating 